\title{
MR Imaging Features of Amyloid-Related Imaging Abnormalities
}

\author{
J. Barakos, R. Sperling, S. Salloway, C. Jack, A. Gass, J.B. Fiebach, D. Tampieri, D. Melançon, Y. Miaux, G. Rippon, R. Black, Y. Lu,
} H.R. Brashear, H.M. Arrighi, K.A. Morris, and M. Grundman

\begin{abstract}
BACKGROUND AND PURPOSE: AD is one of the few leading causes of death without a disease-modifying drug; however, hopeful agents are in various phases of development. MR imaging abnormalities, collectively referred to as amyloid-related imaging abnormalities, have been reported for several agents that target cerebral A $\beta$ burden. ARIA includes ARIA-E, parenchymal or sulcal hyperintensities on FLAIR indicative of parenchymal edema or sulcal effusions, and ARIA-H, hypointense regions on gradient recalled-echo/T2* indicative of hemosiderin deposition. This report describes imaging characteristics of ARIA-E and ARIA-H identified during studies of bapineuzumab, a humanized monoclonal antibody against A $\beta$.
\end{abstract}

MATERIALS AND METHODS: Two neuroradiologists with knowledge of imaging changes reflective of ARIA reviewed MR imaging scans from 210 bapineuzumab-treated patients derived from 3 phase 2 studies. Each central reader interpreted the studies independently, and discrepancies were resolved by consensus. The inter-reader $\kappa$ was 0.76 , with $94 \%$ agreement between neuroradiologists regarding the presence or absence of ARIA-E in individual patients.

RESULTS: Thirty-six patients were identified with incident ARIA-E (17.1\%, 36/210) and 26 with incident ARIA-H (12.4\%, 26/210); of those with incident ARIA-H, 24 had incident microhemorrhages and 2 had incident large superficial hemosiderin deposits.

CONCLUSIONS: In $49 \%$ of cases of ARIA-E, there was the associated appearance of ARIA-H. In treated patients without ARIA-E, the risk for incident blood products was $4 \%$. This association between ARIA-E and ARIA-H may suggest a common pathophysiologic mechanism. Familiarity with ARIA should permit radiologists and clinicians to recognize and communicate ARIA findings more reliably for optimal patient management.

ABBREVIATIONS: $\mathrm{A} \beta=$ amyloid- $\beta$; AD = Alzheimer disease; ARIA = amyloid-related imaging abnormalities; ARIA-E = amyloid-related imaging abnormalities associated with edema or effusion/exudate; ARIA-H = amyloid-related imaging abnormalities associated with hemosiderin deposition

A D remains a leading cause of morbidity and mortality without the availability of a disease-modifying agent. Although the cause of AD remains unclear, the amyloid hypothesis has been a leading postulate on which many therapies have been developed and are now in various phases of clinical evaluation. Several agents specifically designed to lower cerebral $\mathrm{A} \beta$ burden

Received August 9, 2012; accepted after revision December 2.

From the California Pacific Medical Center (J.B.), San Francisco, California; Synarc (J.B., Y.M.), Newark, California; Brigham and Women's Hospital (R.S.), Massachusetts General Hospital, Harvard Medical School, Boston, Massachusetts; Warren Alpert Medical School of Brown University (S.S.), Providence, Rhode Island; Mayo Clinic (C.J.), Rochester, Minnesota; University Hospital Mannheim (A.G.), University of Heidelberg, Mannheim, Germany; Center for Stroke Research (J.B.F.), Charité, Berlin, Germany; NeuroRx Research (D.T., D.M.), Montreal, Quebec, Canada; Pfizer Inc (G.R., R.B.), Collegeville, Pennsylvania; Janssen Alzheimer Immunotherapy Research \& Development, LLC (H.R.B., H.M.A., K.A.M., M.G.), South San Francisco, California; and Global Partners Inc (M.G.), Cambridge, Massachusetts.

This work was supported by Janssen Alzheimer Immunotherapy Research \& Development, LLC, and Pfizer Inc. Michael Grundman was a paid consultant to Janssen Alzheimer Immunotherapy Research \& Development, LLC, in the development of this article. Editorial support was provided by Lisette T. Arnaud, PhD, at Phase Five Communications Inc and was funded by Pfizer Inc and Janssen Alzheimer Immunotherapy Research \& Development, LLC. have been associated with MR imaging abnormalities of the brain. ${ }^{1-4}$ Reported MR imaging findings appear similar among various drugs, despite differences in the proposed mechanisms of action, indicating some commonality in effect. In this report, we specifically describe the imaging abnormalities associated with bapineuzumab, a humanized monoclonal antibody against cerebral $\mathrm{A} \beta$.

Imaging findings reported with bapineuzumab have occurred in both phase 1 and 2 clinical studies and have typically been identified in asymptomatic patients undergoing routine scheduled MR imaging surveillance. ${ }^{1,5}$ These MR imaging findings

Paper previously presented at: Annual Meeting of the American Society of Neuroradiology and the Foundation of the ASNR Symposium, April 21-26, 2012 New York, New York; 12th International Stockholm/Springfield Symposium on Advances in Alzheimer Therapy, May 9-12, 2012; Stockholm, Sweden; and 36th European Society of Neuroradiology Annual Meeting, September 20-23, 2012; Edinburgh, Scotland.

Please address correspondence to Jerome Barakos, MD, California Pacific Medical Center, 2333 Buchanan St, San Francisco, CA 94115; e-mail: barakosmd@gmail.com http://dx.doi.org/10.3174/ajnr.A3500 
Table 1: Clinical characteristics of ARIA

\begin{tabular}{lcccc}
\hline \multirow{2}{*}{$\begin{array}{c}\text { ARIA } \\
\text { Component }\end{array}$} & $\begin{array}{c}\text { Primary Diagnostic } \\
\text { Imaging Sequence }\end{array}$ & $\begin{array}{c}\text { Nature of Leakage } \\
\text { Products }\end{array}$ & \multicolumn{2}{c}{ Location of Increased Vascular Permeability } \\
\hline ARIA-E & FLAIR & Proteinaceous fluid & Vasogenic edema & Leptomeninges \\
ARIA-H & GRE/T2* & Heme products & $\begin{array}{c}\text { Microhemorrhage (hemosiderin } \\
\text { deposits }<10 \mathrm{~mm} \text { or large }\end{array}$ & $\begin{array}{c}\text { Sulcal effusion/exudate } \\
\text { Superficial hemosiderin } \\
\text { deposits }\end{array}$ \\
& & & hemosiderin deposits $\geq 10 \mathrm{~mm})$ & \\
\hline
\end{tabular}

Note:-GRE indicates gradient recalled-echo.

were characteristic of transiently extravasated fluid within the brain parenchyma and were initially described as vasogenic edema among $\mathrm{AD}$ clinical specialists. ${ }^{5}$ With time, a more diverse set of imaging changes was noted that could not be accurately described by the term "vasogenic edema." This spectrum of imaging findings included parenchymal edema and ranged from sulcal effusions to the appearance of associated blood-degradation products. In response to this spectrum of observations, a recent expert workgroup suggested the comprehensive term "ARIA," because the imaging findings encountered are thought to be pathophysiologically related to leakage of intravascular contents due to shifts in amyloid. ${ }^{6}$ The designation "ARIA" has been further subdivided into ARIA-E and ARIA-H (Table 1). ARIA-E has been applied to FLAIR signal abnormalities thought to represent parenchymal edema and/or sulcal effusions (ie, exudates), whereas ARIA-H refers to hemosiderin deposition detected on gradient recalled-echo/T2*-weighted sequences believed to represent blood-degradation products, including microhemorrhages $(<10$ $\mathrm{mm}$ ) and larger superficial hemosiderin deposits ( $\geq 10 \mathrm{~mm}$ ). The purpose of this report was to describe the imaging characteristics of ARIA.

\section{MATERIALS AND METHODS}

This imaging report is derived from the source population of 210 patients with $\mathrm{AD}$ treated with bapineuzumab from 3 phase 2 studies, the methodology of which has been published. ${ }^{7}$ In brief, 2 studies were blinded, randomized clinical trials, whereas the third was an open-label trial in which all patients were eligible for bapineuzumab. Key inclusion criteria consisted of a diagnosis of probable AD with a Mini-Mental State Examination score of 1626. Key exclusion criteria were clinically significant neurologic diseases other than AD. Patients were additionally excluded if they had a screening-visit brain MR imaging scan indicative of $>1$ microhemorrhage or any significant abnormality including but not limited to evidence of a single prior hemorrhage of $>1 \mathrm{~cm}^{3}$, multiple lacunar infarcts, or evidence of a single prior infarct of $>1 \mathrm{~cm}^{3}$. There were no specific exclusionary criteria regarding baseline senescent white matter disease. At initial study enrollment, patients were randomized to bapineuzumab or placebo and received the study drug every 13 weeks for 18 months. Per study protocol, safety MR imaging was performed at baseline and at 13-week intervals, 6 weeks after each infusion. A uniform MR imaging protocol was used in this multisite study and included FLAIR, gradient recalled-echo/T2*, DWI, T2, and 3D T1weighted sequences on $1.5 \mathrm{~T}$ systems. All MR imaging machines were calibrated using the American College of Radiology phantom at baseline and at 4-month intervals according to specifications made by the American College of Radiology MRI Accreditation Program. Unscheduled imaging was also performed as dictated by any significant change in clinical symptoms or as follow-up to monitor and assess ongoing ARIA-E; 2572 MR imaging scans were obtained on the 210 bapineuzumab-treated study participants.

All scans were reviewed and interpreted contemporaneously by local MR imaging readers at each of the 30 clinical study sites in the United States. Subsequently, all examinations were centrally reviewed by 2 neuroradiologists with specific knowledge of the range and spectrum of imaging changes reflective of ARIA. Central readers, blinded with respect to the study arm, placebo or drug, and Apolipoprotein $\varepsilon 4$ status, reviewed all imaging studies in serial order, having baseline and serial imaging available for comparison. Patients were identified as having incident ARIA-E by the detection of new areas of either parenchymal or sulcal FLAIR hyperintensity, in the absence of imaging features that would suggest other pathologies such as ischemia and neoplasm. Readers recorded lesion location (cerebrum versus cerebellum) by hemisphere (right or left) and by lobe involved (frontal, parietal, occipital, and/or temporal). Incident ARIA-H was determined by the detection of any blood products noted as new hypointense lesions on the blood-sensitive gradient recalled-echo/T2* weighted imaging sequence. Incident hypointense lesions were characterized by size and location, namely, whether they were microhemorrhages $(<10 \mathrm{~mm}$ in diameter) or large hemosiderin deposits ( $\geq 10 \mathrm{~mm}$ ). ARIA-H lesion localization was recorded by using a similar map as outlined above. Each central reader interpreted the studies independently, and discrepancies were resolved by consensus. The inter-reader $\kappa$ was 0.76 , with $94 \%$ agreement between neuroradiologists regarding the presence or absence of ARIA-E in individual patients.

\section{RESULTS}

Thirty-six patients were identified with incident ARIA-E (17.1\%, $36 / 210)$, and 26, with incident ARIA-H (12.4\%, 26/210); of those with incident ARIA-H, 24 had incident microhemorrhages and 2 had incident large superficial hemosiderin deposits (Table 2). The most commonly encountered MR imaging feature of ARIA-E at detection was a region of sulcal FLAIR hyperintensity in $78 \%$ (28/36) of cases, referred to as ARIA-E effusion/exudate (Fig 1). Of these, 13 also had some degree of adjacent parenchymal FLAIR hyperintensity, with parenchymal involvement described as ARIA-E edema (Fig 2). Parenchymal hyperintensity without sulcal hyperintensity was observed in 8 patients. Parenchymal involvement $(n=21)$ was always coupled with negative findings on a DWI scan, with resolution of the FLAIR signal on serial imaging (Fig 3). Of the 36 cases of ARIA-E identified by the central readers, $15(42 \%)$ were not identified by the local readers.

Gadolinium was administered in 5 of the 36 patients in whom ARIA-E was detected. In most cases, there was no significant pa-

AJNR Am J Neuroradiol 34:1958-65 Oct 2013 www.ajnr.org 1959 


\begin{tabular}{|c|c|c|c|}
\hline ARIA Type: Incidence & $\begin{array}{l}\text { Location: Isolated } \\
\text { Leptomeningeal }\end{array}$ & Location: Isolated Parenchymal & Location: Combined \\
\hline ARIA-E: 36/210 (17\%) & ARIA-E: sulcal effusion: 15/36 (42\%) & ARIA-E: parenchymal edema: 8/36 (22\%) & $\begin{array}{c}\text { ARIA-E: effusion and edema } \\
\text { combined: } 13 / 36(36 \%)\end{array}$ \\
\hline ARIA-H: 26/210 (12.4\%) & $\begin{array}{l}\text { ARIA-H: superficial hemosiderin } \\
\text { deposit: } 2 / 26(8 \%)\end{array}$ & $\begin{array}{l}\text { ARIA-H: parenchymal microhemorrhage: } \\
24 / 26(92 \%)\end{array}$ & \\
\hline
\end{tabular}
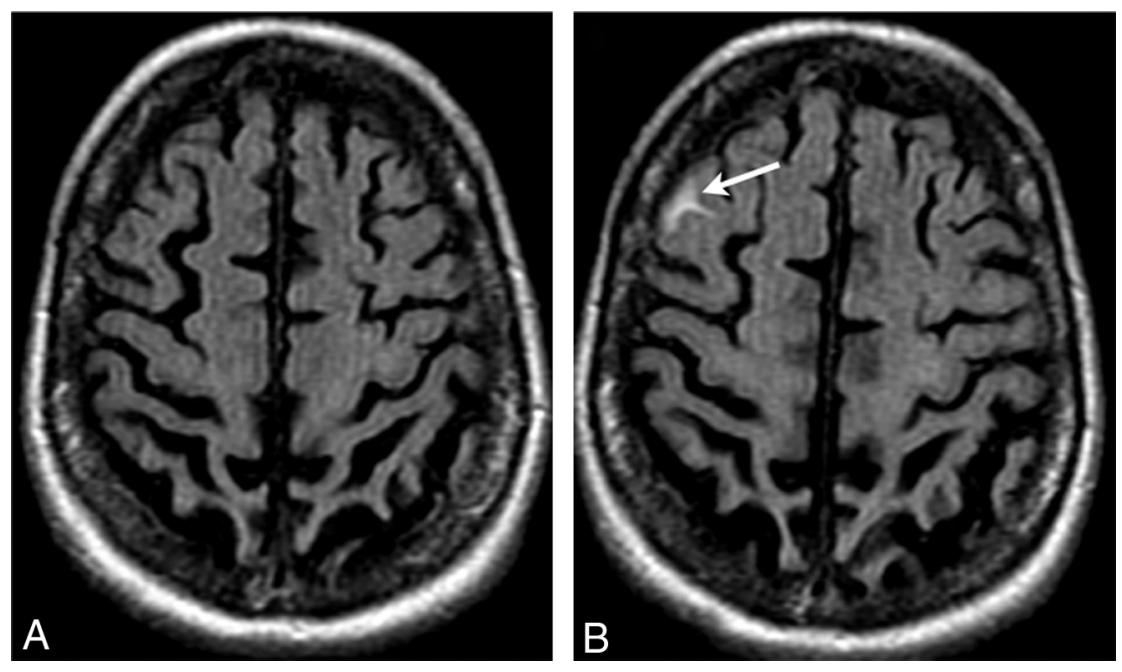

FIG 1. ARIA-E (effusion); sulcal FLAIR hyperintensity. FLAIR MR imaging at baseline (A) and week 19 $(B)$. On the follow-up surveillance scan (week 19), sulcal FLAIR hyperintensity developed in the right frontal region (arrow). No associated signal abnormality was identified on other imaging sequences, including $\mathrm{Tl}$ and gradient recalled-echo/T2* imaging (not shown). Because the imaging appearance mimicked that of a subarachnoid hemorrhage, CT and lumbar puncture were performed, both of which had normal findings, revealing no evidence of blood products.

renchymal enhancement other than a mild prominence in cortical vascular enhancement, as would be expected in any nonspecific parenchymal or leptomeningeal edematous process. Of the patients with ARIA-E, 35 had acceptable gradient recalled-echo/ T2* images at study enrollment for the assessment of incident ARIA-H; of these, 49\% (17/35) had incident ARIA-H microhemorrhages (Fig 4). One patient with ARIA-E did not have an acceptable pretreatment baseline gradient recalled-echo/T2*. However, timing was not always simultaneous, with 24\% (4/17) of incident ARIA-H microhemorrhages identified on an image before first identification of ARIA-E and 29\% (5/17) of incident ARIA-H microhemorrhages identified on an image after the first identification. Seven patients developed incident ARIA-H microhemorrhages without ARIA-E (4\%, 7/174). In 2 other patients with ARIA-E, ARIA-H, as large superficial hemosiderin deposits, also developed during follow-up (Fig 5). Two-thirds (69\%, 25/36) of ARIA-E cases were identified after the first or second bapineuzumab infusion, corresponding to the imaging at study weeks 6 and 19, respectively. Before identification of ARIA-E, the mean number of infusions was 2.4 (range, 1-7; median, 1.7). The actual duration of ARIA-E was difficult to accurately define because imaging was routinely performed at 13 -week intervals. However, occasional unscheduled examinations were performed at shorter intervals to monitor ARIA-E evolution for cases detected during the clinical study. Given these limitations, follow-up imaging revealed ARIA-E at a median duration of 16 weeks.

With ARIA-E edema $(n=21)$, cerebral parenchymal involvement was unilateral in $67 \%(14 / 21)$ and bilateral in 33\% (7/21) of patients. ARIA-E edema was noted in the cerebellum in 4 patients, of whom 2 also had cerebral involvement and involvement in 2 was isolated to the posterior fossa. Lesion severity ranged from subtle to nearly holohemispheric involvement (Fig 4) and, as reported in Sperling et al, ${ }^{7}$ was associated with both Apolipoprotein $\varepsilon 4$ allele status and increasing drug dosage. However, no specific baseline clinical or imaging features were predictive of the development of ARIA. In particular, no correlation was identified between patients with microhemorrhage at baseline and subsequent development of ARIA.

When tabulated by regions of lobar involvement, ARIA-E edema was distributed as follows: occipital lobe, $48 \%$ $(n=10)$; frontal lobe, $43 \%(n=9)$; temporal lobe, $29 \%(n=6)$; cerebellum, $19 \%(n=4)$; and parietal lobe, $14 \%$ $(n=3)$. (Total was $>100 \%[n=21]$ because a single case may have contributed to multiple regions depending on the extent of involvement.)

\section{DISCUSSION}

In several clinical $\mathrm{AD}$ studies with agents directed at modifying cerebral $\mathrm{A} \beta$ burden, including bapineuzumab studies, hyperintense FLAIR signal abnormalities have been detected on brain MR imaging and are often associated with the development of blood products. ${ }^{1,5}$ This constellation of imaging features is in keeping with the hypothesis of altered vascular permeability, ${ }^{6,8}$ which is now recognized as part of the ARIA spectrum. ARIA-E specifically refers to associated parenchymal edema and sulcal effusions, whereas ARIA-H refers to hemosiderin deposits from blood products within the parenchyma or leptomeninges. Although the focus of this report is on imaging features of ARIA associated with therapeutic amyloid-modifying agents, similar imaging findings have been noted to occur spontaneously, such as in cerebral amyloid angiopathy. ${ }^{9-15}$

Therapy-related ARIA should be a diagnostic consideration when specific imaging findings are encountered in the appropriate clinical setting — namely, a patient being treated with an amyloid-modifying agent with exclusion of other underlying pathologies. For the most part, clinical history will be of value as the patient will typically be asymptomatic, imaged as part of routine drug study surveillance (ie, safety scan). 

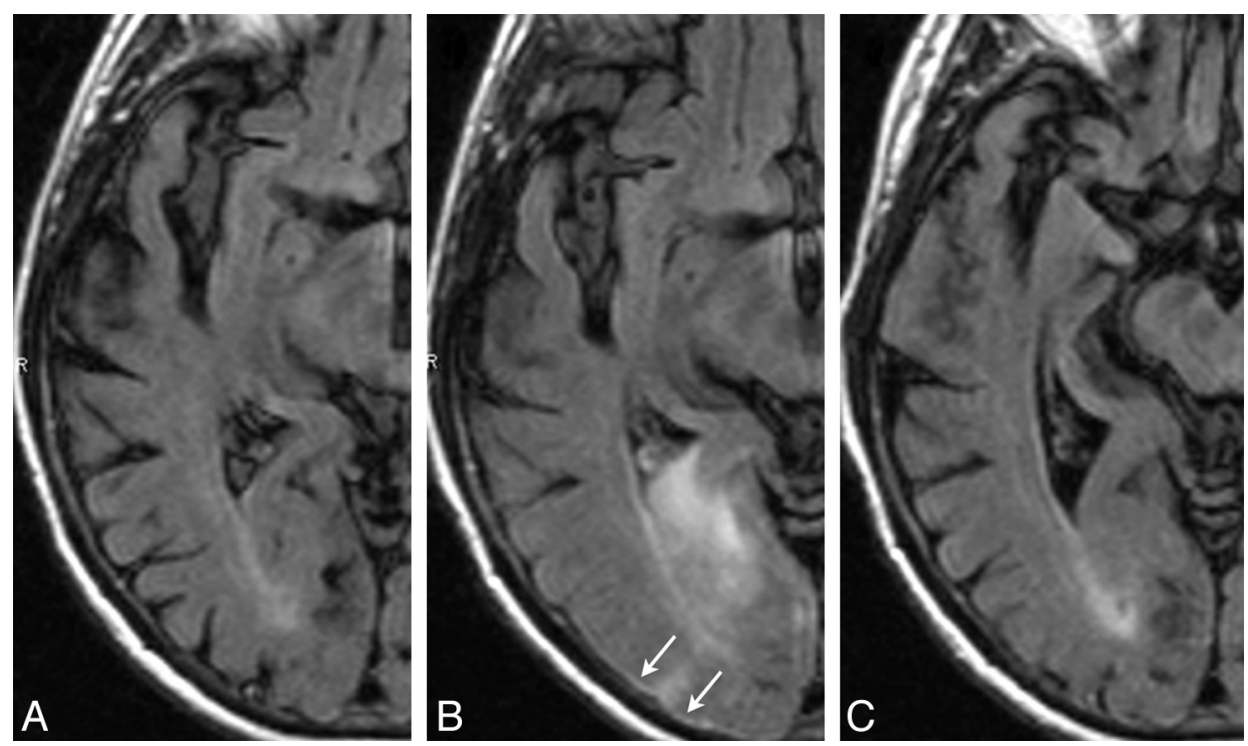

FIG 2. ARIA-E (edema and effusion); parenchymal and sulcal FLAIR hyperintensity. FLAIR sequences at baseline $(A)$, week $19(B)$, and week $32(C)$. By week 19 (6 weeks after the second dose of bapineuzumab), a zone of cortical swelling, sulcal effacement, and parenchymal hyperintensity has developed in the right temporal-occipital lobe. A small sulcal effusion and faint overlying linear leptomeningeal hyperintensity (arrows) help define the area of abnormality. By week 32, these findings have largely resolved. As is typically the case with bapineuzumab-related ARIA, the patient remained asymptomatic throughout all imaging time points.

\section{Pathophysiology}

Potential mechanisms giving rise to ARIA in association with agents that target amyloid removal, including immunotherapy agents such as bapineuzumab, remain to be fully explained. However, both imaging and histopathologic studies have demonstrated that immunotherapies may reduce amyloid burden and are associated with the development of ARIA-type changes. ${ }^{16}$ Additionally, prior reports indicate that the risk for ARIA-E is increased in Apolipoprotein $\varepsilon 4$ allele carriers and is also associated with an increasing dose of bapineuzumab. ${ }^{5}$ These findings are supported by studies indicating that vascular $\mathrm{A} \beta$ accumulation and clearance are involved. ${ }^{8,17}$ Studies in PDAPP mice have shown that the murine version of bapineuzumab (3D6) binds and clears vascular $\mathrm{A} \beta$ in a dose-dependent manner and is associated with a transient increase in vascular permeability. ${ }^{18}$ Hypothetically, bapineuzumab may have a similar action on vascular $A \beta$ in some patients, resulting in transient vascular leakage of fluid, protein, and/or red cells. The suggestion that ARIA is related to increases in vascular permeability is also supported by the frequent co-occurrence of ARIA-E and ARIA-H, albeit not always with simultaneous onset, which indicates that these are likely related phenomena. Specifically, in review of the phase 2 bapineuzumab data, incident ARIA-H was detected in association with $49 \%$ of patients with ARIA-E but in only $4 \%$ of patients without ARIA-E.

Vascular $\mathrm{A} \beta$ burden is highest in the cerebral cortex and leptomeninges, ${ }^{19}$ which may account for the predilection of ARIA to occur in these areas. Also of note are reports of spontaneous ARIA-like phenomena in patients with cerebral amyloid angiopathy, described as cerebral amyloid angiopathy-related inflammation, and in $\mathrm{AD}$ patients without identifiable underlying cerebral amyloid angiopathy on MR imaging. ${ }^{9-15}$ An underlying endovascular inflammatory component has been described in this condition and may provide an additional potential mechanism involved with the ARIA phenomenon. Nevertheless, these findings indicate that shifts in cerebral amyloid accumulation may occur naturally, occasionally resulting in increased vascular permeability or in overwhelmed transport systems and leading to edema and sulcal effusions.

\section{ARIA-E (Edema versus Effusions)}

The parenchymal and sulcal forms of ARIA-E are distinguished by differences in anatomic location (intra-axial versus extra-axial, respectively) but are similar in that both are transient in nature and not associated with restricted (reduced) diffusion abnormalities. The imaging features of ARIA-E edema are consistent with parenchymal edema and presumably reflect leakage of intravascular fluid and proteins into the parenchymal interstitial compartment due to the compromise of vessel-wall integrity.

In contrast to the parenchymal pattern of ARIA-E edema, sulcal FLAIR hyperintensities representing extra-axial effusions/exudate were referred to as ARIA-E effusions. In these cases, findings were consistent with proteinaceous fluid confined to the subarachnoid/leptomeningeal space, with the resulting effect on $\mathrm{T} 1$ and $\mathrm{T} 2$ relaxation yielding a corresponding hyperintensity on FLAIR imaging. Although isolated effusions were noted, especially in the early phases of ARIA-E, progression of these effusions commonly was associated with some degree of contiguous parenchymal FLAIR hyperintensity (ARIA-E edema).

\section{Imaging Considerations}

Given that imaging features of ARIA-E comprised parenchymal edema and/or sulcal proteinaceous effusions, identification was most conspicuous on MR imaging when one used a T2-weighted FLAIR sequence. In addition, sulcal effusions were entirely obscured with proton-attenuation or T2-weighted imaging due to the T2 hyperintensity of CSF. As such, FLAIR imaging is essential in detecting ARIA-E effusions. As expected, even the parenchymal form of ARIA-E was routinely visualized with improved conspicuity by using FLAIR sequences in contrast to proton-attenuation or T2-weighted imaging.

\section{Differentiating ARIA-E from Other Pathologies}

As with routinely encountered parenchymal or sulcal FLAIR hyperintensities occurring in clinical practice, the MR imaging features of ARIA-E are nonspecific and may reflect a wide variety of pathologic conditions. Thus, in the setting of a patient being 

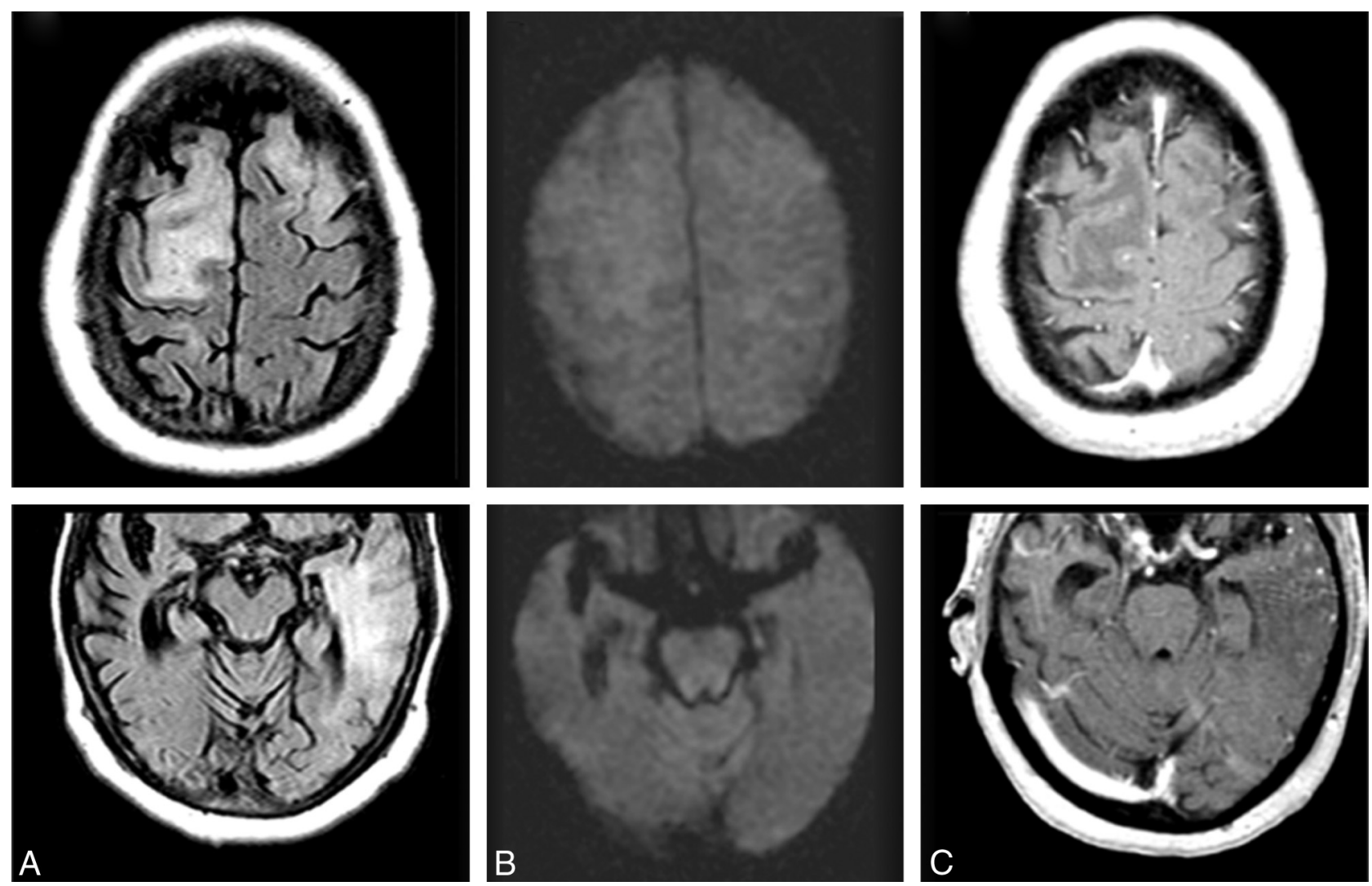

FIG 3. ARIA-E (edema); week 19 parenchymal FLAIR hyperintensity. Week 19 FLAIR ( $A$ ) and corresponding DWI (B) and week 19 gadolinium (C). On the surveillance scan for week $19(A)$, several large regions of parenchymal edema develop, principally in the bifrontal and left temporal regions, which resolve on follow-up (not shown). Week 19 DWI reveals minimal T2 shinethrough (B), but no ADC abnormality (not shown). With gadolinium, there is no frank enhancement with only mild prominence of overlying cortical vasculature, in keeping with nonspecific cerebral swelling (C).

treated with an amyloid-modifying agent, any newly detected region of FLAIR hyperintensity must be fully characterized to exclude other potential pathologies. The parenchymal FLAIR hyperintensity of ARIA-E edema may be mimicked by ischemia, neoplasm/metastatic disease, cerebritis, and so forth. As such, DWI is essential to evaluate ischemia, and gadolinium administration is important in excluding an underlying source of parenchymal edema such as malignancy and infection/cerebritis. In contrast, the leptomeningeal FLAIR hyperintensity of ARIA-E effusion may be mimicked by various conditions including subarachnoid hemorrhage, meningitis, and even inadequate CSFnulling artifacts. ${ }^{20,21}$ In fact, in the first few patients in whom sulcal effusions were encountered, because the appearance of the effusion precisely mimics imaging features of a subarachnoid hemorrhage, CT and lumbar puncture were performed. Such testing consistently confirmed lack of subarachnoid hemorrhage as a source of these imaging findings. Additionally, in several of the initial cases of ARIA-E, gadolinium was administered. These contrast-enhanced MR images typically revealed little to no enhancement, with mild overlying vascular congestion, as expected in any edematous cortical process.

MR imaging abnormalities characteristic of ARIA-E are very similar to posterior reversible encephalopathy syndrome. Both conditions are usually reversible, demonstrating parenchymal and leptomeningeal involvement, with potential for development of blood products. Additionally, both conditions reveal a predi- lection for the occipital lobes, often more symmetric with posterior reversible encephalopathy syndrome. Although isolated leptomeningeal involvement and milder cases seem more common with ARIA-E than in posterior reversible encephalopathy syndrome, this difference is most likely due to matters of timing. Specifically, cases of ARIA-E were detected early in the disease course due to routine surveillance imaging. During this early phase of ARIA-E, leptomeningeal involvement predominates. In contrast, patients identified with posterior reversible encephalopathy syndrome often come to attention because of frank neurologic symptoms and dysfunction, including seizures and coma and thus are at a more severe phase of their disease, with parenchymal involvement overshadowing the leptomeningeal disease. Pathophysiologic underpinnings of increased vascular permeability of both ARIA-E and posterior reversible encephalopathy syndrome are thought to be very similar, thus explaining the parallel imaging features of these conditions.

In summary, ARIA-E should be a highly likely diagnostic consideration when there is evidence of parenchymal edema and/or sulcal FLAIR hyperintensity, identified in patients recently exposed to an amyloid-modifying agent and in whom no evidence of any other inciting cause or underlying lesion can be found.

\section{ARIA-H}

In $49 \%$ of cases of ARIA-E detected on MR imaging, there was the associated appearance of small amounts of blood degradation prod- 

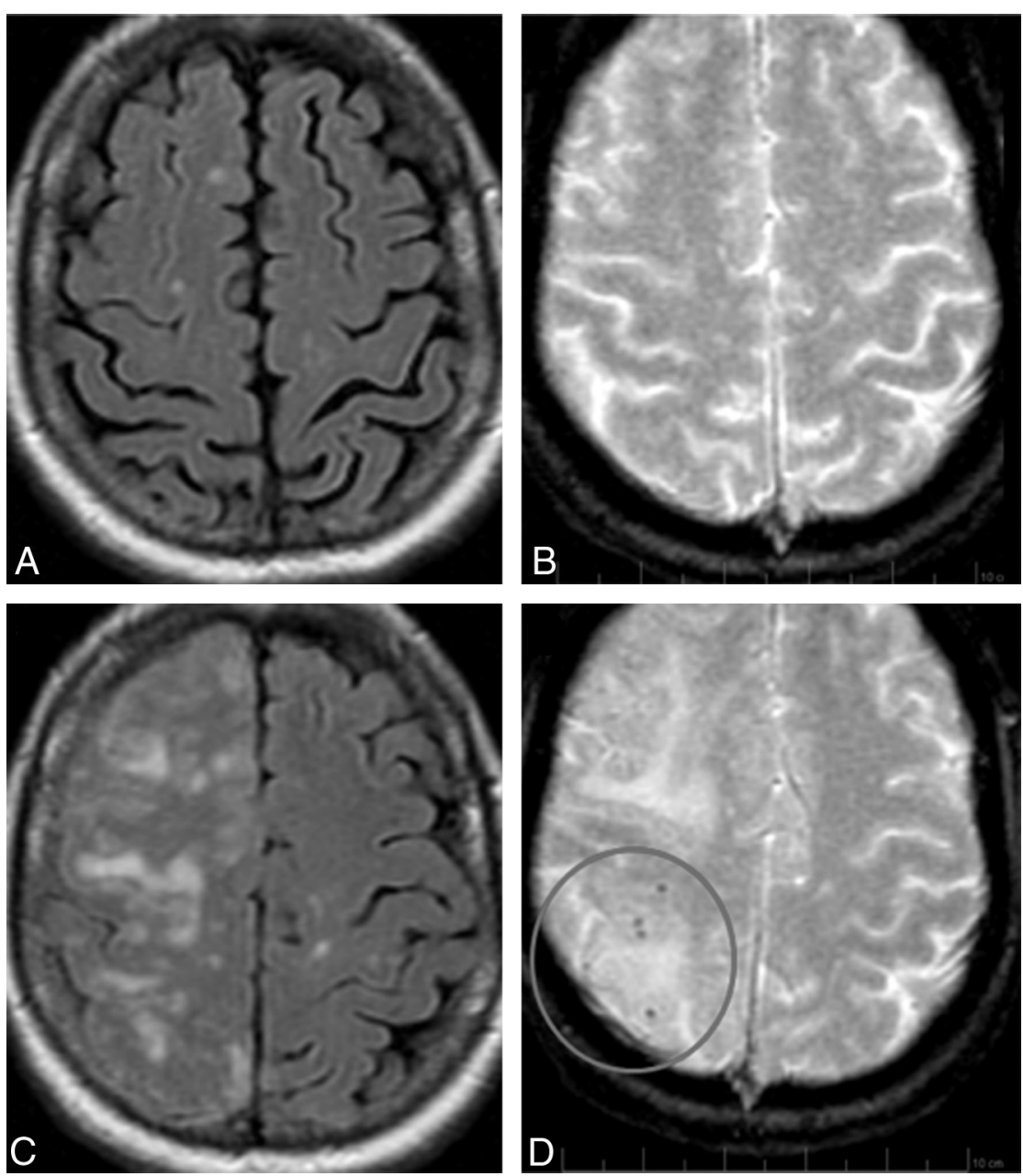

FIG 4. ARIA-E (edema) with incident ARIA-H (microhemorrhages). FLAIR and corresponding gradient recalled-echo/T2* sequences of the same patient, at baseline $(A$ and $B$ ) and week 19 ( $C$ and $D$ ). By week 19 (6 weeks after the second dose of bapineuzumab), significant right-hemispheric edema has developed. As is characteristic of ARIA-E, despite the extensive parenchymal changes, DWI and ADC findings remained normal (not shown), confirming these findings as reflecting vasogenic as opposed to cytotoxic edema. On the corresponding gradient recalled-echo/T2* images, there is concomitant development of several punctate microhemorrhages in the right parietal region (circle). On subsequent imaging, the cerebral edema resolved while the microhemorrhages remained stable (not shown). In general, the severity of ARIA changes was associated with both Apolipoprotein $\varepsilon 4$ allele status and drug dosage. As such, this patient was an Apolipoprotein $\varepsilon 4$ homozygote and in the highest drug-dose arm.

ucts (ARIA-H). In treated patients without ARIA-E, the risk for incident blood products was $4 \%$. Association between ARIA-E and ARIA-H may suggest a common pathophysiologic mechanismnamely, increased vascular permeability to the point at which both fluid and red blood cells cross the vessel wall. These blood products were most commonly microhemorrhages, defined as punctate parenchymal foci of signal void on gradient recalled-echo/ $\mathrm{T}^{*}$-weighted images, measuring $<10 \mathrm{~mm}$ in diameter. Less commonly, larger superficial foci of blood products were detected measuring $\geq 10 \mathrm{~mm}$ in diameter (ie, superficial hemosiderin deposits).

Overall, these blood-degradation products were petechial in nature and, as with microhemorrhages, only identifiable as signal voids on gradient recalled-echo/T2*-weighted images. Without such sequences, these minute amounts of blood product cannot be detected because they are invisible on T1, T2, and FLAIR imaging due to use of rephasing pulse gradients. This feature highlights the importance of using gradient recalled-echo/T2*-weighted sequences for surveillance of ARIA-H.

A review of the phase 2 bapineuzumab data did not identify an associated risk of the presence of microhemorrhages from the pretreatment baseline image with the subsequent development of ARIA-E. However, lack of an association may reflect study enrollment criteria, whereby patients with $\geq 2$ microhemorrhages were excluded. As such, the hypothesis of such a risk requires further evaluation because the observed association of ARIA-E with an increasing Apolipoprotein $\varepsilon 4$ allele number suggests that an increased amyloid burden relating to cerebral amyloid angiopathy underlies various proposed mechanisms in the development of ARIA-E. Both AD and cerebral amyloid angiopathy share a common neuropathology relating to the deposition of $\mathrm{A} \beta$, which affects arterial wall integrity and interference with perivascular drainage pathways. In this regard, it is interesting to note the imaging similarities between cerebral amyloid angiopathy-related inflammation ${ }^{12-15}$ and ARIA-E associated with amyloid-modifying agents. Both conditions may reveal asymmetric, patchy, or confluent FLAIR hyperintense lesions with little or no enhancement after gadolinium administration and with imaging features suggesting noncytotoxic edema on DWI sequences. Additionally, in both conditions, gradient recalled-echo/T2*-weighted images may show associated foci of parenchymal and leptomeningeal microhemorrhages, more consistently in cerebral amyloid angiopathy-related inflammation though a case without microhemorrhages has been proposed. ${ }^{11}$

Given that compromise of both arterial wall integrity and perivascular pathways is a proposed mechanism in the development of ARIA, eliminating patients with frank imaging features of cerebral amyloid angiopathy (eg, evidence of numerous parenchymal or leptomeningeal microhemorrhages) is prudent. Current FDA guidelines for enrolling patients in studies assessing amyloid-modifying agents recommend excluding patients with $\geq 5$ microhemorrhages and any evidence of siderosis or prior parenchymal hemorrhage, all of which are potential imaging features that may be reflective of underlying cerebral amyloid angiopathy.

Given the ARIA imaging features outlined, an ARIA surveillance scanning protocol is recommended. Minimum surveillance imaging for ARIA should consist of FLAIR, gradient recalledecho/T2*, and DWI. FLAIR and gradient recalled-echo/T2* 

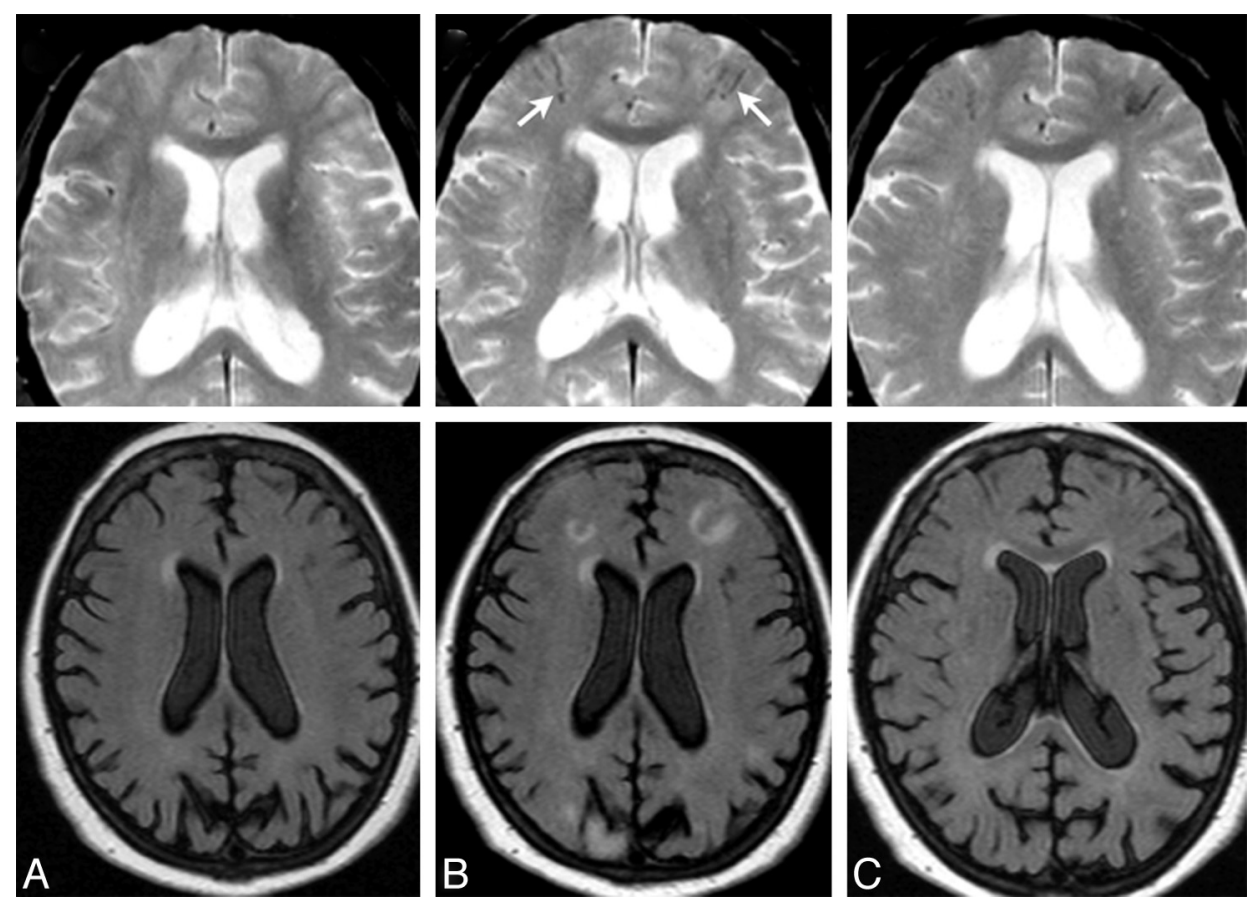

FIG 5. ARIA-E (edema) with incident ARIA-H (superficial hemosiderin deposits). Gradient recalled-echo/ $\mathrm{T}^{*}$ and corresponding FLAIR sequences from the same patient, at baseline $(A)$, week $19(B)$, and week $28(C)$. By week $19(B)$, several regions of parenchymal edema have developed, including bifrontal regions and the right parieto-occipital lobe. On the corresponding gradient recalled-echo/T2* images, by week 19 , there is concomitant development of several superficial linear areas of blood-degradation products (arrows) in the bifrontal regions $(\geq 10 \mathrm{~mm}$ ). By week 28 , the FLAIR changes relating to ARIA-E have resolved, with blood products re-demonstrated.

provide optimal detection of ARIA-E and ARIA-H, respectively. When a new area of parenchymal FLAIR hyperintensity is detected, DWI allows the evaluation of cytotoxic edema. T1 has a limited role, but in clinical trials when acquired by using $3 \mathrm{D}$ technique, it allows hippocampal volumetric calculations. Finally, additional imaging sequences such as gadolinium enhancement should be performed at the discretion of the radiologist when further lesion characterization is needed.

\section{CONCLUSIONS}

A spectrum of MR imaging features has been described and designated as ARIA, with 2 subtypes, ARIA-E and ARIA-H, which are associated with several therapeutic agents directed at modification of amyloid burden in the treatment of AD. Familiarity with these findings will allow MR imaging readers to approach such imaging studies with increased diagnostic sensitivity and specificity, ensuring that treating clinicians are aware of potential changes reflected on imaging studies and allowing them to properly manage drug administration.

\section{ACKNOWLEDGMENTS}

We acknowledge and thank all participating patients and their caregivers, as well as investigators. Additionally, we thank Lorna Fang, Janssen Alzheimer Immunotherapy Research \& Development, LLC, for help in statistical programming.

Disclosures: Jerome Barakos—UNRELATED: Consultancy: Synarc, Janssen, Pfizer, Comments: consultant to Synarc, an imaging clinical research organization that helps manage the imaging aspect of clinical trials for pharmaceutical companies including Elan, Janssen, and Pfizer, Payment for Development of Educational Presentations:
Janssen, Pfizer, Comments: helped in developing educational materials for Medscape on behalf of Janssen and Pfizer. Reisa Sperling-RELATED: Grant: Janssen,* Comments: This work was conducted as part of a phase 2 study; we were a clinical site, Consulting Fee or Honorarium: Janssen, Pfizer, Comments: a paid consultant in the past for Janssen and Pfizer, but not in 2012, Support for Travel to Meetings for the Study or Other Purposes: Janssen, Pfizer, Comments: I have been paid to speak at meetings in 2011, UNRELATED: Consultancy: Merck, Eisai, Satori, Kirin, Roche, Janssen, Pfizer, Comments: consulted for multiple companies developing drugs for AD, Payment for Lectures (including service on Speakers Bureaus): Janssen, Pfizer, Lilly, Comments: in 2011, Payment for Development of Educational Presentations: Janssen, Comments: in 2011, I was paid to help develop educational materials. Stephen Salloway-RELATED: Grant: Janssen Alzheimer Immunotherapy,* Pfizer,* Consulting Fee or Honorarium: Janssen Alzheimer Immunotherapy, Pfizer, Elan, Support for Travel to Meetings for the Study or Other Purposes: Janssen Alzheimer Immunotherapy, Pfizer, Elan, UNRELATED: Consultancy: Bristol-Myers Squibb (BMS), Avid, Baxter, Bayer, Sanofi, Lilly, Grants/ Grants Pending: BMS, ${ }^{*}$ Avid,* Genentech,* Baxter, ${ }^{*}$ GE Healthcare, ${ }^{\star}$ Merck, ${ }^{*}$ Payment for Development of Educational Presentations: Janssen Alzheimer Immunotherapy, Travel/Accommoda-

tions/Meeting Expenses Unrelated to Activities Listed: BMS, Baxter, Bayer, Sanofi. Clifford Jack—RELATED: Grant: National Institutes of Health,* Consulting Fee or Honorarium: Janssen,* Bristol-Meyer-Squibb,* Johnson \& Johnson.* Achim GassRELATED: Consulting Fee or Honorarium: Pfizer/Janssen Advisory Board member Payment for Lectures (including service on Speakers Bureau): Bayer Schering, Biogen Idec, Merck Serono, Novartis, Teva Neurosciences. Jochen B. Fiebach—UNRELATED Consultancy: Wyeth, Novartis, BMS, Comments: central assessment of cerebral MRI examinations, Payment for Development of Educational Presentations: Biolmaging, Comments: introduction in neuroimaging. Donatella Tampieri-RELATED: Consulting Fee or Honorarium: NeuroRx, Comments: consulting in MRI. Yves Miaux-UNRELATED: Consultancy: Synarc. Gregory Rippon—UNRELATED: Employment: Pfizer, Comments: paid employee of Pfizer Inc, Stock/Stock Options: Pfizer, Comments: presently own stock and have received stock options as an employee of Pfizer Inc. Ronald Black-Employment: Pfizer, Comments: full-time employee of Pfizer, a sponsor of this study, Stock/Stock Options: Pfizer, Comments: stock and stock options in Pfizer. Yuan Lu-Employment: Janssen Alzheimer Immunotherapy. H. Robert Bras hear-RELATED: Other: Janssen Alzheimer Immunotherapy, UNRELATED: Employment, Stock/Stock Options, Other: Janssen Alzheimer Immunotherapy, Comments: I am an employee of Janssen Alzheimer Immunotherapy, the cosponsor company who conducted the clinical research studies for which the MR images in the manuscript were obtained and assessed (I also received stock and stock options as an employee). H. Michael Arrighi-RELATED: Other: Janssen Alzheimer Immunotherapy, Comments: employee of Janssen Alzheimer Immunotherapy, a sponsor of the work, UNRELATED: Employment: Janssen Alzheimer Immunotherapy, Stock/Stock Options: Elan Pharmaceuticals, Comments: Elan owns $49.9 \%$ of Janssen Alzheimer Immunotherapy, Travel/Accommodations/Meeting Expenses Unrelated to Activities Listed: Janssen Alzheimer Immunotherapy, Other: Janssen Alzheimer Immunotherapy. Kristen A. Morris—UNRELATED: Employment: I am a Janssen Alzheimer Immunotherapy employee. Michael Grundman-RELATED: Consulting Fee or Honorarium, Payment for Writing or Reviewing the Manuscript: Janssen Alzheimer Immunotherapy, UNRELATED: Consultancy, Employment:Janssen Alzheimer Immunotherapy. *Money paid to the institution.

\section{REFERENCES}

1. Black RS, Sperling RA, Safirstein B, et al. A single ascending dose study of bapineuzumab in patients with Alzheimer disease. Alzheimer Dis Assoc Disord 2010;24:198-203 
2. Sperling R, Bronen R, Greenberg S, et al. Three cases of apparent vasogenic edema (VE) from a phase 2 clinical trial of the gamma secretase inhibitor BMS-708163 in patients with mild-to-moderate AD. Alzheimers Dement 2011;7(suppl):S377

3. Doody R. Results from independent analyses of the EXPEDITION (solanezumab) study data conducted by the Alzheimer's Disease Cooperative Study (ADCS). In: Proceedings of the Annual Meeting of The American Neurological Association, Boston, Massachusetts. October 7-9, 2012

4. Ostrowitzki S, Deptula D, Thurfjell L, et al. Mechanism of amyloid removal in patients with Alzheimer disease treated with gantenerumab. Arch Neurol 2012;69:198-207

5. Salloway S, Sperling R, Gilman S, et al. A phase 2 multiple ascending dose trial of bapineuzumab in mild to moderate Alzheimer disease. Neurology 2009;73:2061-70

6. Sperling RA, Jack CR Jr, Black SE, et al. Amyloid-related imaging abnormalities in amyloid-modifying therapeutic trials: recommendations from the Alzheimer's Association Research Roundtable Workgroup. Alzheimers Dement 2011;7:367-85

7. Sperling R, Salloway S, Brooks DJ, et al. Amyloid-related imaging abnormalities in patients with Alzheimer's disease treated with bapineuzumab: a retrospective analysis. Lancet Neurol 2012;11:241-49

8. Boche D, Zotova E, Weller RO, et al. Consequence of Abeta immunization on the vasculature of human Alzheimer's disease brain. Brain 2008;131(pt 12):3299-310

9. Eng JA, Frosch MP, Choi K, et al. Clinical manifestations of cerebral amyloid angiopathy-related inflammation. Ann Neurol 2004;55:250-56

10. Scolding NJ, Joseph F, Kirby PA, et al. Abeta-related angiitis: primary angiitis of the central nervous system associated with cerebral amyloid angiopathy. Brain 2005;128(pt 3):500-15

11. Carlson C, Estergard W, Oh J, et al. Prevalence of asymptomatic vasogenic edema in pretreatment Alzheimer's disease study co- horts from phase 3 trials of semagacestat and solanezumab. Alzheimers Dement 2011;7:396-401

12. Oh U, Gupta R, Krakauer JW, et al. Reversible leukoencephalopathy associated with cerebral amyloid angiopathy. Neurology 2004; 62:494-97

13. Kinnecom C, Lev MH, Wendell L, et al. Course of cerebral amyloid angiopathy-related inflammation. Neurology 2007;68:1411-16

14. Savoiardo M, Erbetta A, Storchi G, et al. Case 159: cerebral amyloid angiopathy-related inflammation. Radiology 2010;256:323-27

15. Sakaguchi $H$, Ueda $A$, Kosaka $T$, et al. Cerebral amyloid angiopathyrelated inflammation presenting with steroid-responsive higher brain dysfunction: case report and review of the literature. J Neuroinflammation 2011;8:116

16. Rinne JO, Brooks DJ, Rossor MN, et al. 11C-PiB PET assessment of change in fibrillar amyloid-beta load in patients with Alzheimer's disease treated with bapineuzumab: a phase 2, double-blind, placebo-controlled, ascending-dose study. Lancet Neurol 2010;9:363-72

17. Weller RO, Subash M, Preston SD, et al. Perivascular drainage of amyloid-beta peptides from the brain and its failure in cerebral amyloid angiopathy and Alzheimer's disease. Brain Pathol 2008; 18:253-66

18. Schroeter S, Khan K, Barbour R, et al. Immunotherapy reduces vascular amyloid-beta in PDAPP mice. J Neurosci 2008;28:6787-93

19. Vinters HV. Cerebral amyloid angiopathy: a critical review. Stroke 1987;18:311-24

20. Filippi CG, Ulug AM, Lin D, et al. Hyperintense signal abnormality in subarachnoid spaces and basal cisterns on MR images of children anesthetized with propofol: new fluid-attenuated inversion recovery finding. AJNR Am J Neuroradiol. 2001;22:394-99

21. Stuckey SL, Goh TD, Heffernan T, et al. Hyperintensity in the subarachnoid space on FLAIR MRI. AJR Am J Roentgenol 2007;189: 913-21 